\title{
“Théâtre et christianisme" di Charles Mazouer. Per una lettura globale del teatro francese
}

\section{Michele Mastroianni}

\section{(2) OpenEdition}

\section{Journals}

\section{Edizione digitale}

URL: http://journals.openedition.org/studifrancesi/5214

DOI: 10.4000/studifrancesi.5214

ISSN: 2421-5856

\section{Editore}

Rosenberg \& Sellier

\section{Edizione cartacea}

Data di pubblicazione: 1 dicembre 2016

Paginazione: 489-496

ISSN: 0039-2944

\section{Notizia bibliografica digitale}

Michele Mastroianni, «"Théâtre et christianisme" di Charles Mazouer. Per una lettura globale del teatro francese », Studi Francesi [Online], 180 (LX | III) | 2016, online dal 01 janvier 2018, consultato il 18 septembre 2020. URL : http://journals.openedition.org/studifrancesi/5214 ; DOI : https://doi.org/ 10.4000/studifrancesi.5214

\section{(c) (i) (9)}

Studi Francesi è distribuita con Licenza Creative Commons Attribuzione - Non commerciale - Non opere derivate 4.0 Internazionale. 


\section{"Théâtre et christianisme" di Charles Mazouer. Per una lettura globale del teatro francese ${ }^{1}$}

\section{Abstract}

Charles Mazouer's essay on Théâtre et christianisme analyzes the complex - partially harmonious and partially conflicting - relationship between French theatre and religion, from medieval mystères to the revival of a religious theatre in the Twentieth century. The analysis is particularly focused on Sixteenth and Seventeenth centuries. In these centuries, on the one hand there is the christianization of the Greek and Latin theatre, and on the other hand there is the secularization of the medieval religious theatre. It is a process that gives birth to modern theatre, with its mixing of different theological currents, philosophies and cultural sources.

Charles Mazouer, che in cinque monumentali volumi (pubblicati tra il 1998 e il 2014) ${ }^{1}$ ha offerto la storia dettagliata del teatro francese dal Medioevo alla fine del Seicento - strumento oggi irrinunciabile per tutti gli studiosi della letteratura teatrale -, raccoglie una parte considerevole dei frutti di una vita consacrata a indagare la drammaturgia (quarantatré saggi composti tra il 1980 e il 2013)² in un volume che, oltre a fornire studi a volte difficili da reperire, appresta nuove chiavi interpretative del fenomeno drammaturgico, a partire dalla produzione medievale fino a quella dell'âge classique. Infatti, non soltanto i saggi proposti sono rivisti e aggiornati sul piano bibliografico e sottoposti a una attualizzazione per quanto concerne gli argomenti, ma si creano introduzioni e passi di collegamento che conferiscono coerenza a un discorso profondamente unitario.

La prospettiva è quella del rapporto col fatto religioso, che nel teatro francese - come nel teatro di quasi tutte le culture - è elemento originario e fondatore. Nel nostro caso, evidentemente, si tratta del rapporto col cristianesimo, dalla cui liturgia il teatro trae primo alimento, della cui teologia si fa portavoce, ma con la cui dottrina (anche per lo svilupparsi di una doppia filière, religiosa e profana) può entrare in rotta di collisione suscitando censure e, soprattutto, dando spunto a una ricca produzione controversistica sia polemica sia apologetica.

Proprio per la nascita religiosa del teatro quale estensione della liturgia (peraltro concepita anch'essa, spesso, come mise en dramaturgie della memoria scritturale o del contenuto di fede), il genere del mystère religieux acquista una centralità nel volume di Mazouer, che offre una delle riflessioni più ampie e acute che siano state concepite su questa forma teatrale. La cui influenza e popolarità è tale che, nel cuore del Rinascimento, quando l'ossessione umanistica per l'antico trova nella volontà di fare rinascere il teatro greco e latino una delle sue espressioni più significative, i mystères

(1) CH. Mazouer, Le Théâtre français du Moyen Âge, Paris, Sedes, 1998; ID., Le Théâtre français de la Renaissance, Paris, Champion, $2002\left(2013^{2}\right)$; ID., Le Théâtre français de l'âge classique. I: Le premier $\mathrm{XVII}^{\mathrm{e}}$ siècle, Paris, Champion, 2006; ID., Le Théâtre français de l'âge classique. II: L'apogée du classicisme, Paris, Champion, 2010; ID., Le Théâtre français de l'âge classique. III: L'arrière-saison, Paris, Champion, 2014.

(2) CH. Mazouer, Théâtre et christianisme. Études sur l'ancien théâtre français, Paris, Champion («Convergences» 2), 2015. 
in quanto genere, lungi dallo scomparire sotto l'onda del disprezzo (qual è quello, per esempio, ostentato dalla Pléiade) per una letteratura lontana dai nuovi ideali estetici, si trovano al centro di una polemica che culmina, sul piano istituzionale, nell'editto di interdizione del 1548, ad opera del Parlamento di Parigi. In realtà, malgrado l'editto parigino sia seguito da documenti analoghi emessi da parlamenti di provincia, noi troviamo sopravvivenze tenaci dei mystères, e non soltanto negli influssi di linguaggio o di tematica presenti in quel teatro religioso di struttura classica che prende piede nel Rinascimento in parallelo al teatro profano d'imitazione greco-latina, ma in una vera e propria ripresa del genere mystère, sia con funzione apologetica nelle "missioni" antiprotestanti sia con funzione parenetica quale veicolo di formazione popolare. Nella sua indagine puntuale Mazouer dimostra l'importanza di uno spoglio di documenti trascurati in genere dagli storici del teatro quali, per esempio, sono le cronache delle missioni e della predicazione: nella fattispecie, una lettura dei cronisti cappuccini della missione antiprotestante nel Chiablese (1597-1598) permette di ricostruire un repertorio di mystères rappresentati spesso nel quadro di celebrazioni liturgiche (o paraliturgiche) come le Quarantore. Nelle Quarante-Heures di Thonon (1598), la pia pratica, nata a Milano nel 1547 ed estesa a tutta la Chiesa alla fine del Cinquecento in chiave antiprotestante a difesa della dottrina cattolica dell'Eucarestia, vede l'introduzione di un vero e proprio mystère (sulla caduta della manna dal cielo) fra le ore di adorazione eucaristica e le processioni. Si tratta di una pratica corrente, quella appunto di «faire une représentation de quelque dévote histoire pour attirer les peuples» (cit. a p. 180) in un'alternanza con i momenti di preghiera. E interessante peraltro notare come la sopravvivenza dei mystères nelle missioni popolari si verifichi malgrado le reticenze manifestate dalla Chiesa nei confronti del teatro religioso, di quello almeno di massa, e soprattutto malgrado una legislazione contraria. In realtà, si riconosceva al teatro una forza rappresentativa dei contenuti dottrinali, spesso astratti, della predicazione e della catechesi, tale da rendere la forma teatrale (proprio per il suo impatto emozionale) canale privilegiato di trasmissione della verità religiosa.

Queste osservazioni sulla sopravvivenza dei mystères, al di là sia delle condanne istituzionali sia della svolta rinascimentale foriera della nascita di un teatro nuovo, si ampliano in una serie di riflessioni sul permanere (o sul rinascere) in epoca moderna di un teatro religioso che riprende la struttura del genere medievale, in parte sull'onda di quel gusto estetizzante per il Medioevo che si sviluppa nelle letteratura e nell'arte fin-de-siècle e primonovecentesca e caratterizza il Simbolismo europeo, ma soprattutto nel quadro di un dibattito sul teatro che mira al rinnovamento della scena dell'epoca, di cui si denuncia a volte l'elitismo di un certo Simbolismo a volte la sciatteria e banalità del Boulevard. In tale contesto, la volontà di fare un teatro popolare (vedi Le Théâtre du peuple di Romain Rolland) trova un archetipo nel mystère medievale, tanto più che l'impresa, per certi versi archeologica, seppure fortemente interessata al sociale (si pensi all'esperienza di Copeau al Vieux-Colombier), ha come promotori scrittori di dichiarata militanza cattolica o perlomeno d'ispirazione cristiana, come Ghéon, Péguy, Claudel, Milosz e Ghelderode. Questa ripresa del genere medievale è peraltro un fenomeno di portata limitata e, soprattutto, estremamente disuguale nei risultati che vanno dagli esiti felici di alcuni autori di genio come Claudel (ben lontani, nella loro ricerca di un linguaggio nuovo, dai modelli ispiratori) alla platitude della maggior parte della produzione di tale indirizzo, quella di Ghéon per esempio (giustamente Mazouer evoca il théâtre de patronnage). Tuttavia, il discorso sul mystère medievale e sulle sue propaggini moderne è interessante come testimonianza, in Francia, di una riflessione sul teatro visto sotto due aspetti, quello della destinazione popolare e quello dell'ispirazione religiosa.

D'altronde Mazouer, sempre nella prospettiva dell'ispirazione religiosa, parte ancora dal riferimento ai mystères per indagare alcuni testi del teatro del Novecento 
ove teologia della storia e teologia morale offrono spunti tematici che potrebbero in qualche modo ricordare sia la tradizione agiografica sia la tradizione della moralité come sottofondo della drammaturgia. Sarebbe il caso dei testi di Péguy e di Claudel. Quest'ultimo autore in particolare, definendo «mystère en quatre actes et un prologue» l'Annonce faite à Marie, impone un confronto con i mystères del Quattro e Cinquecento. Si tratta di un confronto rivelatore di un superamento della maniera medievale nella direzione dell'approfondimento psicologico e soprattutto nell'impiego di una sottigliezza teologica del tutto estranea alla semplicità, o meglio alla semplificazione catechetica, dei testi antichi. Se è vero, d'altronde, che nel teatro del Novecento (Mazouer sviluppa su Mauriac un ampio discorso che offre strumenti interpretativi del teatro religioso della modernità in generale) una precisa teologia trova espressione drammatica - quella della miseria dell'uomo, del mistero del male e del peccato, contrapposta a una teologia della grazia, che si concretizza in una meditazione sulla santità, sulla purezza e sull'amore - questa teologia ha una precisa illustrazione nell'indagine psicologica, per non dire psicanalitica.

Il complesso discorso sul mystère, in quanto genere che occupa un'indubbia centralità, non sempre avvertita, nella storia del teatro francese, soffermandosi sulle reazioni contro la rappresentazione sacra di espressione popolare (anche se di remota origine paraliturgica), in particolare sulla condanna del 1548 e sulla sua controversa ricezione, mette in luce alcune contraddizioni nelle vicende di un teatro concepito all'origine come forma di edificazione religiosa e come una sorta di pedagogia nell'ambito della fede e della morale. Infatti, proprio a partire da un teatro che si vuole edificante nasce contestazione e censura contro un genere di espressione considerato contrario alla religione e ai valori che essa vuole imporre. Il fatto è che oggetto delle condanne da parte dell'autorità religiosa e civile è il "riso", ovvero la produzione comica e satirica giudicata eversiva e fomite di empietà e d'immoralità in genere. Ora, per certo vi è un théâtre du rire - la farsa, la sottie - che tratta anche le cose di religione in modo critico e parodico. In particolare, Mazouer nella sua riflessione sul tema della contestazione e censura nel teatro si sofferma su quel secolo (14501550) che vede parallela la fioritura di un teatro che si vuole edificante e un teatro che affonda le radici nella festa carnevalesca e nello spirito di un mondo alla rovescia che attraverso il burlesco e il grottesco intende rispecchiare degrado e profanazione dei valori stabiliti ${ }^{3}$. Tuttavia, accanto a un teatro comico costruito esplicitamente contro l'istituzione e il fatto religioso - o perlomeno derisorio nella rappresentazione di questo fatto, al fine precipuo di far ridere -, abbiamo in un teatro edificante, che tratta della salvezza dell'uomo tramite la meditazione sulla vita di Cristo o dei santi, scene realistiche ambientate in un sottobosco sociale (quello della taverna, per esempio, come nel Jeu de Saint Nicolas di Jean Bodel), popolato di ubriaconi, giocatori d'azzardo, imbroglioni, prostitute, o anche solo di sprovveduti o babbei. Il riso viene dunque accolto nei mystères con lo scopo - pur esso pedagogico - di introdurre un momento di distensione nel corso di rappresentazioni lunghe e sfibranti, mediante la messa in evidenza di personaggi ridicoli e di elementi scatologici o mediante una degradazione del tono apportata dal grottesco.

Questo riso nella Chiesa, però, può spesso diventare - come nella farsa, e per influsso di quest'ultima - un riso contro la Chiesa. La parodia del sacro ha un grande

(3) CH. Mazouer, Théâtre et carnaval en France jusqu'à la fin du XVI siècle, «Revue d'Histoire du Théâtre», 1983/2, pp. 147-161; J. Delumeau, Les mentalités religieuses à travers les farces, les sotties et les sermons joyeux (XV'-XVI siècle), in La Piété populaire au Moyen Âge, «Actes du $99^{\circ}$ Congrès national des Sociétés savantes: Besançon, 1974», Paris, Bibliotèque Nationale, 1977, t. I, pp. 181-195. 
rilievo nella tradizione della farsa. Le osservazioni di Mazouer, riassumendo anche con chiarezza gli studi degli ultimi decenni sul carnevalesco, sulla pietà popolare, sulla dialettica fra questa pietà e la pietà istituzionale, hanno il merito di evidenziare il ruolo della parodia e della derisione per quanto concerne il rapporto contrastato tra 'profano' e 'religioso' nel teatro. La rappresentazione farsesca, sottolinea Mazouer (p. 152), «ne se contente pas de mettre en scène des pécheurs; elle invente des situations comiques qui moquent et défigurent le christianisme, qui mettent en cause des aspects centraux des croyances et pratiques chrétiennes par le moyen de la parodie». Questa parodia colpisce i sacramenti (si pensi al tema della falsa confessione, ricorrente anche nella novellistica), le preghiere e i sermoni, la liturgia nei suoi aspetti più venerabili. La Chiesa, pertanto, costretta a tollerare un teatro profano che rovescia il suo ordine e vilipende le cose sante, di fronte a un teatro religioso, quello dei mystères, che concede spazio sempre più largo al comico (e alla derisione) finisce col reagire duramente, tanto più che le élites intellettuali e sociali, a partire dalla svolta rinascimentale, prendono le distanze dal 'riso' popolare. Con la legislazione ostile che ha origine nel divieto parlamentare del 1548 - legislazione peraltro, come si è detto, frequentemente disattesa - inizia quel divorzio fra la Chiesa, il teatro e il 'riso' che sarà confermato nelle lunghe polemiche del Seicento non solo contro il teatro comico ma contro il teatro in genere, considerato fomite di passioni e ostentazione pubblica di empietà.

Una vera querelle (nel secolo delle grandi querelles letterarie e ideologiche) è quella sul teatro, che culmina nello scontro Bossuet/Caffaro ma ha alle spalle la lunga stagione dei débats animati sia dai giansenisti sia dal partito dei dévots. ${ }^{4}$ Mazouer, nel quadro delle discussioni del Seicento, si sofferma su alcuni momenti fondamentali e mette anzitutto in luce come sussista un théâtre de dévotion sulle scene del secolo: in particolare, tra il 1630 e il 1650 Tristan l'Hermite, Rotrou, Du Ryer, Corneille compongono tragedie ispirate alla Bibbia o all'agiografia; dopo il 1650 il teatro d'argomento biblico-cristiano subisce un'eclissi, ma alla fine del secolo questo teatro avrà una ripresa di alto livello con Esther e Athalie di Racine (senza contare una produzione di pièces sacre di autori minori, come Boyer, Campistron, Duché de Vancy, l'abbé de Brueys).

Interessanti sono le linee che Mazouer segue nella sua perlustrazione. In particolare, nell'indagare il rapporto religione/teatro, egli mette in evidenza l'intertestualità fra una manualistica di devozione e una scena teatrale ridotta a finalità di catéchèse dévote (per usare una felice espressione di Marc Fumaroli). Ruolo centrale, fra i testi edificanti del Seicento, occupa La Cour sainte del Padre Nicolas Caussin, vera miniera di pii exempla, concernenti peraltro príncipi e nobili, alla cui classe, seguendo una pedagogia squisitamente gesuita, l'autore si rivolge. A questa fonte attingono i drammaturghi per trarne eroi morali che appartengano rigorosamente all'aristocrazia - un'aristocrazia celebrata come soggetto precipuo di una virtù cristiana praticata secondo le categorie dell'eroismo. D'altronde alla pedagogia gesuita è legato un teatro tragico latino a destinazione scolastica, come pure della pedagogia gesuita - e degli exempla di Caussin - sono debitori tragediografi quali Tristan o Corneille. In particolare, si apre un discorso concernente il teatro religioso in quanto tale, che indaga la presenza del soprannaturale (e alcune grande tematiche cristiane, quali la Provvidenza) nella tragedia secentesca, in Rotrou per esempio. Emerge anche

(4) Cfr. L. Thirouin, L'aveuglement salutaire. Le réquisitoire contre le théâtre dans la France classique, Paris, Champion, 1997; P. Nicole, Traité de la comédie (et autre pièces d'un procès du théâtre), édition critique par L. Thirouin, Paris, Champion, 1998. Cfr. anche J. Dubu, Les églises chrétiennes et le théatre (1550-1850), Grenoble, Pug, 1997. 
la complessità delle problematiche che pone la prospettiva religiosa nell'approccio al teatro. Di teatro 'cristiano', infatti, si può parlare a diverso titolo, non solo per la presenza di una tragedia sainte nel senso proprio, ma anche per l'influsso dei grandi débats dottrinali (o perlomeno della catechesi e predicazione da essi dipendenti): è il caso tipico del problema della predestinazione, immesso da Corneille nella fabula mitologica antica (in Edipe, 1149-1185), con un'interpretazione che si adegua alla prassi pastorale seguita dai direttori di coscienza, alla luce di una teologia di stampo suareziano.

Tuttavia, proprio la lettura di Corneille da parte dei moralisti giansenisti, come Nicole, e degli avversari del teatro in genere, in particolare nel decennio 1661-1671, è significativa di una vera e propria querelle che inizia appena compare la grande edizione del Théâtre del 1660. Mazouer ricostruisce con precisione questa querelle, distinguendo i diversi discorsi critici e repertoriando le accuse, che si riassumono fondamentalmente nel rimprovero di offrire uno spettacolo di «héros livrés à la passion» (Conti), inoltre ripercorre le repliche di Corneille ai suoi accusatori, attraverso le quali emerge una fenomenologia delle passioni, la cui rappresentazione può essere foriera di «vertus morales et politiques, et quelques-unes même chrétiennes» (Corneille). Contrapponendo così una morale (quella dell'agostinismo, di cui massimi esponenti sono i giansenisti, ma non solo) - per la quale è estremamente pericoloso trasformare in spettacolo, anche a fine edificante, qualsiasi passione o vizio - e una morale più ottimista nei confronti della natura umana, Mazouer indica il teatro di Corneille come esemplare dei rapporti religione/teatro, concludendo che le pièces di Corneille «peuvent bien passer pour des illustrations d'un théâtre "modéré chrétiennement", selon les vœux des jésuites favorables au théâtre, où, plus ou moins indirectement, sont proposées les valeurs d'une éthique chrétienne, comme ne l'ont pas vu ses adversaires augustiniens» (p. 446).

La grande querelle sulla drammaturgia si concentra nel Seicento sul teatro comico, bersaglio principale dei moralisti. Il ruolo di Molière, per le polemiche suscitate da L'École des femmes, da Tartuffe e da Dom Juan, è confermato in un'analisi che passa in rassegna gli avversari del grande commediografo e le loro posizioni. Tuttavia Mazouer mette in luce le contraddizioni dei detrattori della commedia, i quali, richiamandosi «à la tradition de l'Église tirée des conciles et des saints Pères» (Conti), sembrano ignorare la realtà del teatro loro contemporaneo, soprattutto la reale essenza di una comicità che rimane loro estranea. D'altra parte gli avversari della commedia non rappresentano la totalità di quel mondo religioso che si interroga sulla moralità del teatro. In particolare, per quanto concerne Molière, Mazouer offre un quadro pieno di contrasti dei lettori che valutano le sue commedie da una prospettiva che si vuole 'cristiana'. Per certo, con una disamina accurata delle tematiche morali della commedia molieriana (e del contesto religioso in cui possono essere recepite), giunge a concludere che Molière rifiuta fondamentalmente l'ordre de l'Église, in quanto «ne s'en prend pas seulement à la cabale des dévots, défendant un espace privé, un espace laïc contre le pouvoir sacerdotal étouffant. En réalité, à interpréter de bon biais son théâtre, on s'aperçoit que Molière propose un univers fort étranger au christianisme» (pp. 484-485). Nello stesso tempo, però, repertoriando i difensori di Molière - gesuiti, per la maggior parte, ma anche personalità lontane dalla loro spiritualità, come Boileau - fa emergere la complessità del discorso morale sul teatro e, soprattutto, evidenzia come il rapporto teatro/cristianesimo, proprio in un secolo (il Seicento) di massima fioritura della produzione teatrale e di intensi dibattiti religiosi, sia una realtà sfuggente, difficile da definire. Il grande merito, qui, del discorso di Mazouer è di aprire piste nuove di lettura e di stabilire prospettive parallele sulla storia del teatro e sulla storia della spiritualità, che vanno al di là delle ricerche degli ultimi decenni, peraltro ricche e puntuali, sulla querelle del teatro in Francia. 
In questa storia, ricostruita da Mazouer, dei rapporti tra teatro e cristianesimo - rapporti che evolvono profondamente, da periodi di armonia a periodi di aspra conflittualità - possiamo considerare centrali i capitoli consacrati alle interferenze delle diverse filières culturali che confluiscono nell'elaborazione del teatro moderno, in quel crogiuolo soprattutto rappresentato dall'Umanesimo, quando un teatro cristiano di tradizione secolare - mystères e moralités - non si sottrae all'inderogabile norma rinascimentale dell'imitazione del classico ${ }^{5}$, assumendo in questa ossessione imitativa non soltanto le forme ma anche i contenuti (i miti, la storia civile) della tragedia greca e latina e nello stesso tempo, sempre per la volontà di fare rivivere la tragedia antica, proiettando sulle tematiche e sugli argomenti cristiani, in primis sugli argomenti biblici, spunti concettuali proprî della produzione drammaturgica classica, considerata esemplare, al punto di accettare, incorporandola, la filosofia tragica dei greci, del tutto incompatibile con la filosofia cristiana. A giusto titolo Mazouer lo sottolinea:

Quand les dramaturges protestants du XVI ie siècle se mirent à écrire des tragédies bibliques, trois régimes philosophiques et religieux, au fond, risquaient d'entrer en interférence: leur foi chrétienne, la théologie biblique propre à l'Ancien Testament où ils allaient chercher leurs sujets, la philosophie tragique qu'impliquait forcément la forme littéraire imitée (p. 390).

Uno degli elementi costitutivi del tragico antico è l'interrogativo sull'atteggiamento dell'uomo di fronte all'ineluttabilità della colpevolezza, della sofferenza e della morte. I tragediografi greci e latini vi rispondevano con una riflessione sui temi della rivolta e dell'accettazione. Questa riflessione è ripresa dalla tragédie sainte rinascimentale alla luce sia della Bibbia sia della teologia cristiana, dato che la rivolta e l'accettazione sono un luogo teologico in cui possono ritrovarsi tanto il credente ebreo quanto il credente cristiano ${ }^{6}$. È nella tragédie sainte pertanto, cui gli studi più recenti (sulla scorta spesso dei lavori di Mazouer) hanno dedicato un'attenzione primaria, che ritroviamo in massimo grado questo intrecciarsi di régimes philosophiques et religieux, nel trasformarsi strutturale dei mystères sul modello classico. In particolare, «on est frappé par la persistance, dans les tragédies bibliques, d'une vision plutôt grecque; il n'est pas très étonnant que le Dieu des armées, le Dieu vengeur ressemble passablement, quand il châtie ses ennemis, au dieu méchant des Grecs» (p. 398). Non soltanto nella tragédie sainte, comunque, ma nel teatro tragico in genere del Rinascimento avviene la sovrapposizione dei due livelli di pensiero - quello classico greco-latino e quello biblico-cristiano - con un processo di cristianizzazione che tocca profondamente la fabula antica.

Il processo avviene in primo luogo sul piano lessicale, a partire dalle prime traduzioni-rifacimento rinascimentali di tragedie classiche: il fatto stesso che questi rifacimenti si presentino come traduzioni, dimostra una volontà di fedeltà all'originale, ma ciò non toglie che siano segnati da forti slittamenti di senso nella direzione della

(5) Ricordiamo che il più antico trattato di drammaturgia della Francia rinascimentale, De l'Art de la Tragédie, del 1572 (pubblicato criticamente in J. DE LA TAIlle, Saül le furieux - La Famine, ou les Gabeonites, éd. par E. Forsyth, Paris, Didier - Stfm, 1968, pp. 2-16), premesso come introduzione al Saül le furieux di La Taille, propone, in riferimento alla tragédie sainte in quanto genere, come modelli normativi Sofocle, Euripide e Seneca $(\ll[\ldots]$ autant de Tragedies $[\ldots .$.$] et autres$ jeux qui ne sont faicts selon le vray art, et au moule des vieux, comme d'un Sophocle, Euripide et Seneque, ne peuvent estre que que choses ignorantes, malfaites, indignes d'en faire cas, et qui ne deussent servir de passetemps qu'aux varlets et menu populaire, et non aux personnes graves», ibid., p. 8.

(6) Cfr. anche CH. Mazouer, Révolte et consentement dans les tragédies bibliques de la Renaissance, in La Tragédie Sainte en France (1550-1610). Problématiques d'un genre, sous la direction de M. Mastroianni, Paris, Classiques Garnier (sous presse). 
cristianizzazione, sul piano anzitutto del lessico, quando mots-clé della lingua classica greca e latina vengono resi con termini appartenenti al vocabolario teologico cristiano (per esempio, charité, piété, péché, forfait, mondanité, abuz, vanité, biens eternelz, bien de basse terre, ecc. ${ }^{7}$. Ma vi è un altro livello, quello della reinterpretazione in senso cristiano della fabula antica nel suo insieme. Valga solo l'esempio dell'Hippolyte di Robert Garnier, riscrittura della tragedia euripidea, in cui il mito pagano fornisce materiale agiografico per creare, sulla falsariga della storia del biblico Giuseppe insidiato dalla moglie di Potifar, l'exemplum edificante di un giovane che perde la vita per salvaguardare la castità e non macchiarsi del peccato d'adulterio (peccato, peraltro, trattato nella prospettiva della morale cristiana $)^{8}$.

Nella sezione centrale del suo lavoro (cap. X: Théologie, pp. 361-406) Mazouer affronta il problema di questa sovrapposizione di piani nella tragedia francese del secondo Cinquecento, partendo dalla considerazione che «dans la mesure où les dramaturges s'abreuvaient beaucoup à la source grecque et reprenaient les sujets antiques, il n'avaient pas conscience que la fable dramatique véhiculait aussi, invariablement, une pensée, une philosophoe, une théologie fort incompatibles avec leur christianisme» (p. 361). Così, attraverso la lettura parallela di due tragedie di Garnier - una tragedia classica d'argomento mitologico (Hippolyte, 1573) e una tragédie sainte d'argomento biblico (Les Juifves, 1583) - viene fatto un confronto fra una teologia del Fato (connessa alla fede in divinità di cui ci si domanda se siano buone o malvage) e una teologia della Provvidenza (connessa alla fede in un Dio unico, il Dio dell'Alleanza, poi il Dio dell'Amore), proponendo questo interrogativo e questa conclusione, che mette in crisi una definizione di tragico ricorrente (Festugière, Steiner, ecc.):

En quoi et dans quelle mesure le tragédies bibliques de la Renaissance, méditant sur l'omniprésente et multiforme souffrance, illustrent-elles le sens tragique de la vie proposé par les tragiques grecs et leur imitateur latin Sénèque, ou s'en libèrent-elles? Car, si l'on veut bien admettre qu'il n'y a qu'un seul tragique au monde, le tragique grec, il ne peut y avoir, à la vérité, de tragique biblique, ni de tragique chrétien; la Providence divine judéo-chrétienne devrait les congédier radicalement (p. 326).

In una repertoriazione di tematiche che possono rappresentare, in questo gioco di sovrapposizioni fra pagano e cristiano, corrispondenze per quanto concerne una concezione di fondo che possa essere definita "tragica", Mazouer comincia con l'esplorare i grandi assi del motivo della collera di Dio nelle tragedie bibliche (ventisei su una trentina di pièces fra 1550 circa e 1610), motivo di cui studia la funzionalità drammaturgica e la portata teologica. Per contro, nella lettura contrastiva di Hippolyte e Les Juifves, di cui si è detto, si dimostra che la sovrapposizione di piani ideologici, favorita anche da continui slittamenti semantici, finisce col creare un modello coerente di tragedia rinascimentale, che peraltro ha come fattore di resa unitaria il riferimento al trascendente:

(7) Cfr. M. Mastroianni, Le "Antigoni” sofoclee del Cinquecento francese, Firenze, Olschki, 2004; ID., Slittamenti semantici nelle traduzioni di tragedie classiche del Cinquecento francese: la cristianizzazione, in Elaborazioni poetiche e percorsi di genere. Miti, personaggi e storie letterarie, a cura di M. Mastroianni, Alessandria, Edizioni dell'Orso, 2010, pp. 529-545; Le syncrétisme pagano-chrétien à l'époque de l'Humanisme et de la Renaissance, «Actes du Colloque international organisé à Chambéry le 16 et 17 mai 2002 par le C.E.F.I.», publiés sous la direction de S. Lardon, «Franco-Italica» 25-26 (2004), pp. 183-198.

(8) Cfr. D. Cecchetti, Dalla tragedia classica alla tragédie sainte: evoluzione di un linguaggio, in La Tragédie Sainte en France (1550-1610) cit. (sous presse). 
Pour l'essentiel Garnier développe assurément deux théologies. Le vieux mythe grec l'entraîne à la vision pessimiste d'un univers soumis au destin, à la vieille fatalité tragique, où les dieux sont plus vindicatifs que justes, insensibles ou méchants. Dix ans après Hippolyte, Les Juifves nous font sortir du cercle de feu du tragic grec par l'affirmation d'une Providence juste et bonne, par la foi dans le Dieu d'Israël et de Jésus-Christ. Cette théologie correspondait seule aux convictions du croyant Garnier, l'autre leur étant contradictoire. Le dramaturge en eut sans doute l'intuition, qui s'efforça de tempérer un peu le tragique d'Hippolyte, mais sans grand succès (p. 389).

Così, il discorso sui rapporti fra teatro e cristianesimo si rivela in tutta la sua complessità. Soprattutto per l'influsso che in ogni epoca esercitano modelli considerati normativi, ma sempre lontani e appartenenti a tradizioni culturali differenti. Proprio per la ricchezza delle esemplificazioni e la capacità di cogliere i problemi con uno sguardo globale, dominando problematiche diversissime, il lavoro di Mazouer appare uno strumento di lavoro straordinario, foriero di suggerimenti fecondi.

Michele Mastroianni Università del Piemonte Orientale - Vercelli 